\title{
Stability in a Long Length NbTi CICC
}

\author{
L. Bottura, M. Ciotti, P. Gislon, M. Spadoni, P. Bellucci, L. Muzzi, S. Turtù, A. Catitti, S. Chiarelli, \\ A. della Corte, E. Di Ferdinando
}

\begin{abstract}
A crucial issue for a superconducting coil in order to be safely used in the magnetic system of a fusion reactor is stability against all foreseen disturbances. To simulate the fusion machine conditions, including off-normal events, e.g. plasma disruptions, the energy deposition has to be spread over a "long length" Cable In Conduit Conductor (CICC) and a background magnetic field is needed. We have therefore designed and built an experiment consisting of an instrumented NbTi test module inserted in a pair of co-axial pulsed copper coils. A $0.6 \mathrm{~m}$ diameter superconducting coil provides a background magnetic field up to $3 \mathrm{~T}$.

Calibration of the energy inductively coupled between the pulsed coils and the module has been obtained measuring the system temperature increase just after the pulse by means of thermometers positioned along the conductor.

Stability vs. operating current $I_{o p}$ has been examined for different helium temperatures and different background magnetic fields.

The finite element code Gandalf for the stability and quenching transients analysis in forced flow cooled superconducting coils has been run to check the matching with the experimental results.
\end{abstract}

Index Terms-Fusion magnets, CICC, Quench evolution, Stability experiment.

\section{INTRODUCTION}

$\mathrm{O}$ ne of the main problems for the proper design of the superconducting magnets for new generation fusion machines is the ability to predict their stability versus all foreseen electromagnetic disturbances during normal and offnormal working conditions. Numerical codes have been developed to predict the behavior of forced flow cooled superconducting magnets in the hostile environment of a fusion machine. The only way to check the accuracy of the codes is to benchmark against experiments where the stability of an instrumented forced flow magnet is measured as a function of all the relevant thermophysical parameters.

An experiment has been set-up at ENEA, aimed at:

- measuring the critical energy of a "long length" Cable In Conduit Conductor (CICC) under conditions as close as possible to those of a Tokamak machine during the worst event, i.e. a plasma disruption;

- monitoring all the relevant thermophysical parameters

Manuscript received September 18, 2000.

P. Bellucci, A. Catitti, S. Chiarelli, M. Ciotti, A. della Corte, E. Di Ferdinando, P. Gislon, L. Muzzi, M. Spadoni, S. Turtù are with the Associazione EURATOM-ENEA sulla Fusione, Centro Ricerche Frascati, Via E. Fermi 45, 00044 Frascati, Rome, (e-mail: author @ frascati.enea.it).

L. Bottura, CERN, LHC Division, CH 1211 Geneva 23, Switzerland during a quench of the magnet;

- comparing the results of the experiment with the simulations obtained by the thermo-hydraulic predictive code Gandalf [1].

\section{EXPERIMENTAL SET-UP}

The experimental arrangement, shown in Fig.1, is composed of:

1) a $0.6 \mathrm{~m}$ bore superconducting magnet providing a background magnetic field up to $3 \mathrm{~T}$;

2) a NbTi solenoidal winding module, made with a fully instrumented $35 \mathrm{~m}$ long CICC;

3) a pair of pulsed $\mathrm{Cu}$ coils, energized by a capacitor bank and magnetically coupled to the module, to deposit energy into the conductor, up to the transition to the normal state. The maximum discharge voltage is $1500 \mathrm{~V}$, and the capacity is $6.6 \mathrm{mF}$, corresponding to more than $1 \mathrm{~T}$ in the CICC module volume.

The experimental solenoidal module is instrumented with three Ruthenium Oxide sensors soldered on the jacket in the module central part, three sensors for the inlet and outlet pressures, two flow meters and fifteen voltage taps, to monitor the transition and its evolution. The thermometers are positioned, with respect to the helium inlet, at around 14,17 , and $20 \mathrm{~m}$.

The conductor, formed with $3 \times 4 \mathrm{NbTi}$ strands, has a $\mathrm{Cu}$ :nonCu ratio of $5.75,38 \%$ void fraction, a $1.25 \mathrm{~mm}$ thick $\mathrm{CuNi}$ jacket. The solenoid is wound in a double layer configuration. Two power supplies have been used: a linear one, $\pm 5 \mathrm{~V}, 750 \mathrm{~A}$ and a switching one, $\pm 25 \mathrm{~V}, 1000 \mathrm{~A}$, able to keep the output current constant, by means of a feedback circuit, during the external disturbance.

The higher output voltage allows also to follow the quench evolution after a module transition. On the other hand, it produces a very high noise level, so that, if not strictly necessary, the linear power supply was used.

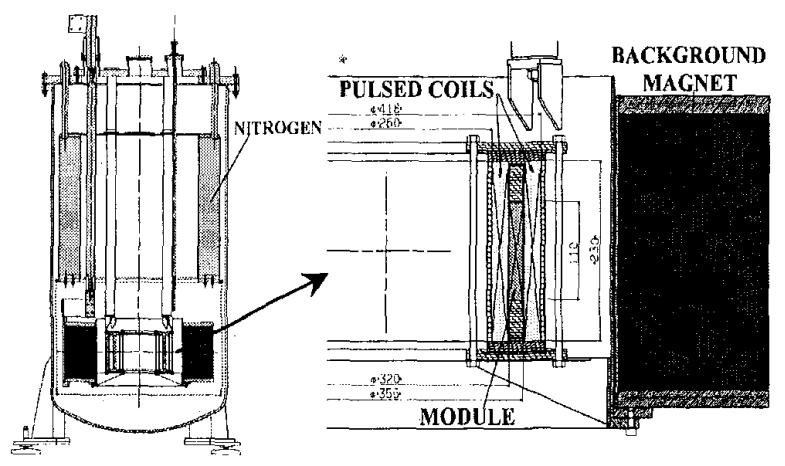

Fig. 1. The experimental arrangement. 


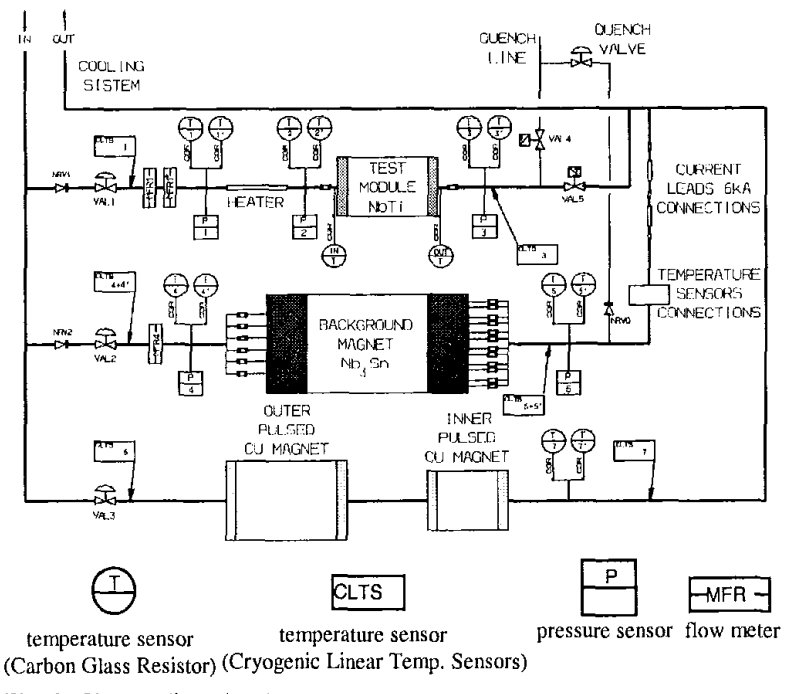

Fig. 2. The cooling circuit.

The cooling circuit (Fig. 2) is also fully instrumented.

The current leads are cooled by a separate circuit fed by an external liquid helium storage.

All sensors' data are acquired with a frequency in the range $1-10 \mathrm{kHz}$, depending on the time resolution needed.

\section{ANALYSIS OF THE EXPERIMENTAL CONDITIONS}

\section{A. Flow Conditions and Temperature Profile}

A small cross section conductor was chosen to limit the operating current below $1000 \mathrm{~A}$. This, together with the conductor long length, leads to a low hydraulic conductance. In spite of a large pressure gradient between the conductor ends ( 10 bar), the helium flow is very low, below the sensitivity of the flow meters. This configuration produces a relevant temperature gradient between helium inlet and outlet, which forces the superconductor transition to happen at the conductor first meters. This occurs, although in the experimental configuration the background magnetic field is lower in the conductor initial part.

To reconstruct the temperature profile along the conductor we used the temperature measured by the three internal thermometers and extrapolated the inlet and outlet temperatures assuming that the helium enthalpy is constant along the conductor length. This assumption is quite accurate, as we measured that the heat input in the central part of the module, with stagnant helium, is negligible.

\section{B. Pulse Duration}

The current pulse that energizes the pair of copper magnets producing the e.m. disturbance is asymmetric in time. It raises approximately linearly, with a time duration of about $30 \mathrm{~ms}$, and decays exponentially with a time constant $(\tau)$ of the order of $90 \mathrm{~ms}$. Assuming a $2 \tau$ criterion, a current pulse duration of about $200 \mathrm{~ms}$ can be evaluated.

Power generation in the conductor is mainly due to the coupling losses and follows a $(\mathrm{dB} / \mathrm{dt})^{2}$ dependence. As an input for the computer code simulations, such a time dependence has been taken. This makes a relevant difference with respect to a symmetric pulse shape, because the power generation is nearly all concentrated in the first $30 \mathrm{~ms}$, i.e. during the current ramp up.

The thermodynamical transformation inside the conductor, as simulated by Gandalf, is affected by this time duration.

This time duration has to be compared to the one expected during a disruption of the magnetic configuration in a Tokamak machine. Even if the plasma current quench has a duration of the order of tens of ms (approximately $1 \mathrm{~ms}$ per MA of plasma current), the time scale on the reactor poloidal and toroidal magnets is different, and it is determined by the exponential decay of the induced currents on the machine vacuum chamber. Thus, pulse duration and shape are not exactly defined, but typical values are, in any case, of the order of hundreds of ms.

\section{Long/short Conductor Length}

The sub-size module is representative of a real scale fusion machine magnet only if it is long enough to simulate the behavior of such a magnet. A typical way to classify long or short length magnets [1] is based on the time the pressure wave takes to propagate through half of the conductor length. If this time is longer than the external pulse duration, the long length assumption is fulfilled. This definition is fully correct, but it turns out to be over-conservative for our module. In fact, due to the low hydraulic conductance (high $\mathrm{L} / \mathrm{d}$ ratio), the helium mass transport caused by the pressure wave at the transition is very slow. On the contrary, due to the high pressure at the inlet, the sound speed in the helium is of the order of $100-250 \mathrm{~m} / \mathrm{s}$. Thus, assuming the quench to start at half of the conductor length, where the background field has a maximum, a module should be around $80 \mathrm{~m}$ long to be defined as a "long length".

The results of the simulation code Gandalf show that the pressure in the conductor decays in a time of the order of a few seconds (Fig. 3).

This suggests us a different criterion for the discrimination between long/short length conductors. A conductor can be said to act as a long length conductor, with respect to external e.m. disturbances, when the thermodynamic transformation to which the helium is submitted during the energy release is isochoric.

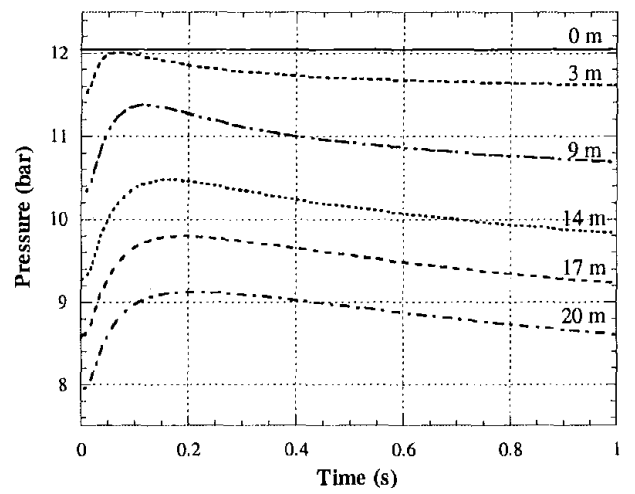

Fig. 3. Pressure vs. time along the conductor, after the magnetic disturbance is applied, as calculated by Gandalf code. 
This assures a different behavior, with respect to the short length conductor, where the thermodynamic transformation is substantially isobaric.

In this experiment, and in general for sub-size long length modules, the differences between the two definitions are considerable.

In our case, having a double layer magnet winding, i.e. with two maxima of the background magnetic field respectively at $1 / 4$ and $3 / 4$ of the conductor length, the literature definition leads to a length of the order of $160 \mathrm{~m}$.

On the contrary, looking at the results obtained with the computer code Gandalf, even at only nine meters from the helium inlet, the pressure relief is about $10 \%$ of the maximum increase after $500 \mathrm{~ms}$ (Fig. 3). Therefore, the long length assumption is completely valid.

\section{Calibration}

As a first step, it was necessary to measure the energy released into the conductor, at zero background field and zero transport current, during the external e.m. disturbance. As explained in Sec. III A, due to the low helium flow, the installed flow meters were out of their measuring range. This made it more difficult to proceed with the calibration of the energy released into the module. The usual calorimetric method couldn't be applied and we had to develop a modified calorimetric approach.

The three Ruthenium Oxide thermometers, positioned inside the module, measure the jacket temperature; we checked, either by using the code Gandalf and by means of a simple 1-D thermal model, that the helium and jacket temperatures reach the equilibrium within a time of the order of $1 \mathrm{~ms}$, while jacket and the external insulation Glass Epoxy have a time shift of the order of $20 \mathrm{~ms}$.

As Fig. 4 shows, at the pulse end the helium temperature reaches its maximum and then starts to decrease, due to the reduction of the pressure, hindered by the low hydraulic conductance.

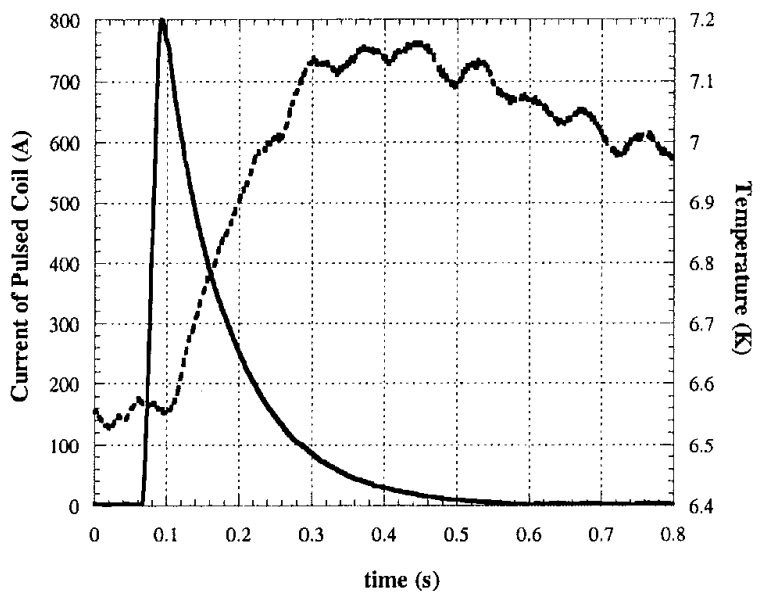

Fig. 4. Typical time evolution of the temperature inside the module after the current pulse is discharged.

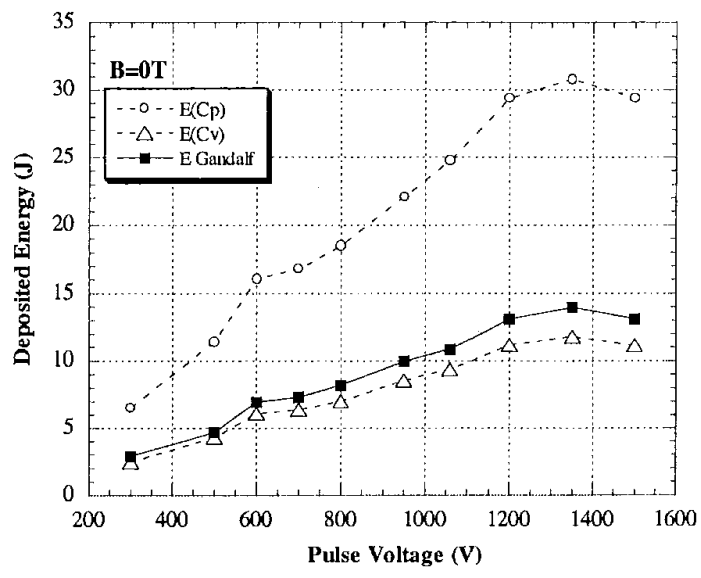

Fig. 5. The energy released as computed by Gandalf and as calculated assuming a perfect isochoric or perfect isobaric thermodynamic transformation.

The time constant of the temperature decrease is of the order of some seconds, i.e. much longer than the time necessary to reach an equilibrium temperature in the system (strands, helium, jacket, glass epoxy).

Therefore, by measuring after a few hundreds of $\mathrm{ms}$ the temperature increase produced by the external e.m. disturbance, it is possible to calculate the energy deposited in the module as a function of the external pulse intensity. The results are shown in Fig 5, where the deposited energy is plotted versus the capacitor bank power supply voltage. The resulting energy, obtained by the code, is compared to the energy that can be calculated assuming a perfect isochoric or perfect isobaric thermodynamic transformation. The comparison shows that in our experimental configuration the constant volume assumption is fulfilled.

\section{EXPERIMENTAL RESULTS}

\section{A. Critical Current Measurements}

Critical current measurements have been performed at different temperatures $(7.2 \mathrm{~K}<\mathrm{T}<7.9 \mathrm{~K})$ and background magnetic fields $(0.1,1.35,2.25 \mathrm{~T})$. As already mentioned in Sec. III $A$, the strong temperature gradient along the conductor forces the module to quench near the helium inlet.

The temperature assumed for the transition zone is the one calculated from the isoenthalpic extrapolation, as explained in Sec. III $A$. The measured data can be fitted with the following expression [2]:

$I_{c}\left(B_{0}, T_{0}\right)=\frac{I_{c 0}}{B_{0}}\left(\frac{B_{0}}{B_{c 2}\left(T_{0}\right)}\right)^{\alpha}\left(1-\frac{B_{0}}{B_{c 2}\left(T_{0}\right)}\right)^{\beta}\left(1-\left(\frac{T_{0}}{T_{c 0}}\right)^{1.7}\right)^{\gamma}$ with $T_{\text {co }}=9.2 \mathrm{~K}$

The fit includes also the $4.2 \mathrm{~K}, 3 \mathrm{~T}$ point measured on a single strand. The fit has been obtained through an optimization on the $I_{c 0}$ normalization coefficient and $\gamma$ parameter, while making a parametrical variation alternatively on the $\alpha(0.6<\alpha<0.8)$ and $\beta(0.8<\beta<0.9)$ parameters. 
The magnetic field on the module has been computed by the computer code Toska, taking into account both the external background magnet and the module self field.

The best fit has been obtained with $\alpha=0.8, \beta=0.8, \gamma=2.45$, in agreement with values reported in literature [2].

The differences between the measured and calculated data are within $20 \%$. The error bar in the fitting does not allow to ascertain if cabling has produced some critical current degradation; on the other hand, the experimental apparatus did not allow a direct measurement around $4.2 \mathrm{~K}$.

\section{B. Stability Measurements}

Stability measurements have been performed once a reference temperature profile has been chosen and the selected transport current supplied. The external e.m. pulse has been fired, its intensity increased up to the value that induced the magnet quench. Measurements have been repeated for different temperature profiles, transport currents (up to $1000 \mathrm{~A}$ ), and background magnetic fields (up to $2.25 \mathrm{~T}$ ) (Figs. 6, 7).

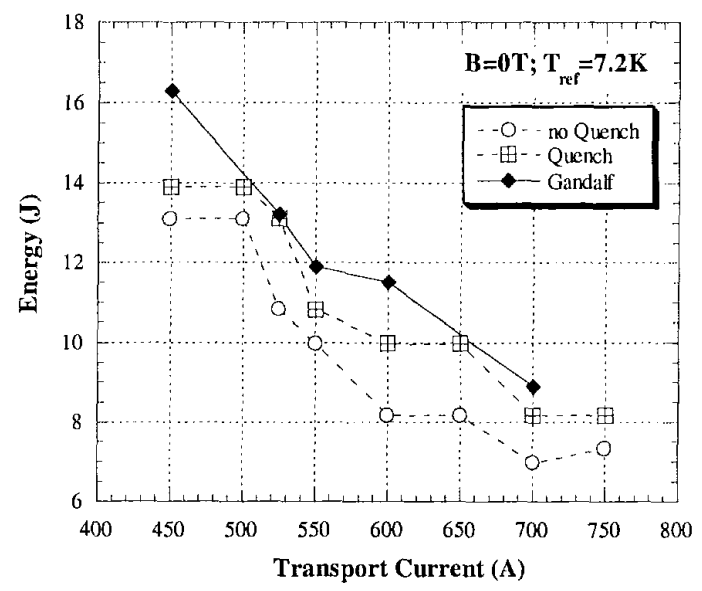

Fig. 6. Stability matgins at $\mathrm{B}=0 \mathrm{~T}, \mathrm{~T}_{\mathrm{ref}}=7.2 \mathrm{~K}$. The values computed by Gandalf code are compared with the experimental measurements.

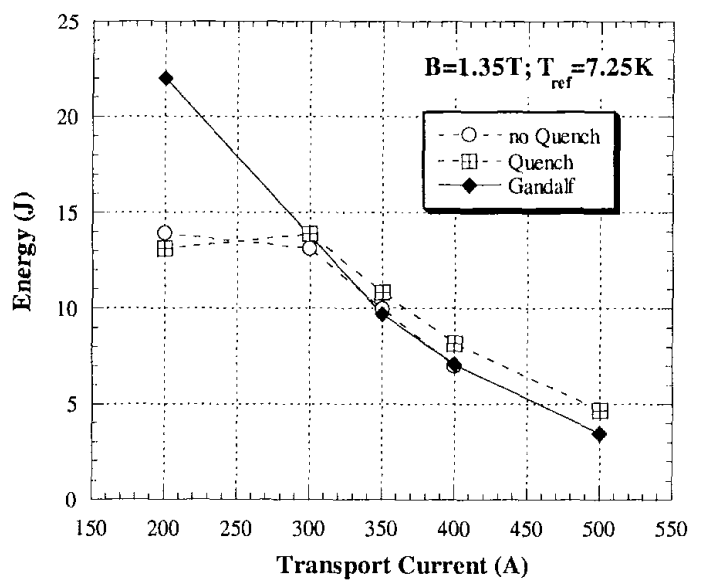

Fig. 7. Stability margins at $\mathrm{B}=1.35 \mathrm{~T}, \mathrm{~T}_{\text {ref }}=7.25 \mathrm{~K}$. The values computed by Gandalf code are compared with the experimental measurements.
For these measurements the $1000 \mathrm{~A}$, switching power supply has been used, because it assures a roughly constant current in the module during the pulse. At the same time this power supply is extremely noisy and all the voltage taps are strongly disturbed, thus preventing to detect where the transition starts. We determined the overall module transition by means of the internal thermometers.

Data have been compared to the results obtained with the computer code Gandalf, as shown in Figs.6,7. The agreement between the experimental and the calculated stability curves is quite good [3].

According to the code, the transition is produced by the combined effect of the magnetic field increase generated by the pulse and by the AC loss heating of the conductor. It starts in the first meters, since the higher temperature is not sufficiently counterbalanced by the lower background magnetic field. In these conditions, the long length assumption is not completely fulfilled in all the experimental runs. Changes in the experimental set-up are foreseen to recover the long length condition.

\section{CONCLUSIONS}

The energy released onto a sub-size NbTi superconducting magnet during an external e.m. disturbance has been evaluated by a modified calorimetric technique. Beside all the difficulties in performing measurements representative of a long length conductor, a full characterization of the magnet has been achieved. A new definition for long/short length conductor experiments is proposed and amply discussed. Critical currents and stability have been measured and compared with the results from the computer code Gandalf, obtaining a good agreement with the experimental data. Care has to be used in planning sub size experiments with respect to the temperature gradient along the conductor, the He mass flow inside it, and the background magnetic field configuration along the module.

Moreover, in order to perform a representative experiment for a fusion magnet, it must be taken into account that the reference pulse duration is not the plasma current quench time, but the decay time of the induced currents in the vacuum vessel and other external structures of fusion machines.

\section{ACKNOWLEDGMENTS}

The authors thank L. Verdini for the development of the data acquisition program, E. P. Balsamo for his electronic suggestions, M. V. Ricci for magnetic calculations, L. Mazza for electronic support.

\section{REFERENCES}

[1] L. Bottura, M. Perrella, J. Basecq, M. Spadoni, A. Martinez., "Stability experiment on long lengths of cable-in-conduit superconductors", $17^{\text {th }}$ Symp.of Fus. Tech., vol. 1, pp. 797-801, September 1992.

[2] L. Bottura, "A practical fit for the critical surface of NbTi", $16^{\text {th }}$ int. Conf. On Mag. Tech., Ponte Vedra Beach, Fl, USA, 1999.

[3] P. Bellucci, M. Ciotti, P. Gislon, M. Spadoni, L. Bottura, L. Muzzi, S. Turtù, "Comparison between the predictions of the thermo-hydraulic code Gandalf and the results of a long length instrumented $\mathrm{CICC}$ module experiment", CHATS Y $2 K$, Frascati, September 2000, submitted for publication. 